Annals of International Medical and Dental Research

E-ISSN: 2395-2822 | P-ISSN: 2395-2814

Vol-8, Issue-1 | January-February 2022

DOI: $10.53339 /$ aimdr.2022.8.1.22

Page no- 168-174 | Section- Research Article (Anaesthesiology)

\title{
Transmuscular Quadratus Lumborum Block for Postoperative Analgesia in Laparoscopic Colorectal Surgery
}

\author{
Sumita Mohanty ${ }^{1}$, Sarat Chandra Jayasingh ${ }^{2}$, Asit kumar Sethi ${ }^{3}$, Sidharth Sraban Routray ${ }^{*}$
}

\begin{abstract}
${ }_{1}^{1}$ Associate Professor, Department of Anaesthesiology, Pain and Palliative care, Acharya Harihar Postgraduate Institute of Cancer, Cuttack, Odisha, India.

Email: jasashree@gmail.com

Orcid ID: 0000-0002-5200-8229

${ }^{2}$ Associate Professor, Department of Surgical Gastroenterology, SCB Medical College, Cuttack, Odisha, India.

Email: Saratchandra818@gmail.com

Orcid ID: 0000-0002-7672-9717

${ }^{3}$ Associate Professor, Department of Surgery, Bhima Bhoi Medical College and Hospital, Balangir, Odisha, India.

Email: asitkumarsethi2021@gmail.com

Orcid ID: 0000-0002-9611-1498

${ }^{4}$ Associate Professor, Department of Anaesthesiology and critical care, S.C.B. Medical College, Cuttack, Odisha, India.

Email: drsidharth74@gmail.com

Orcid ID: 0000-0002-9992-438X

*Corresponding author
\end{abstract}

Received: 01 September 2021

Revised: 30 October 2021

Accepted: 12 November 2021

Published: 22 December 2021

\begin{abstract}
Background: Transmuscular Quadratus Lumborum Block (TQLB) is a newer modality for postoperative pain management. But, its efficacy after laparoscopic colorectal surgery is little researched. The aim of our trial was to access the analgesic efficacy of TQLB in colorectal surgery. Methods: This study was done in 64 patients posted for colorectal surgery who were divided into two groups of 32 each. TQLB was given bilaterally in group RQ with $20 \mathrm{ml}$ of $0.375 \%$ ropivacaine and in group SQ with $20 \mathrm{ml}$ saline. Patients were operated under general anesthesia and were examined for pain at different time points postoperatively. Time required for first analgesic demand was our primary endpoint. Secondary endpoints were total rescue analgesia (paracetamol) required in $24 \mathrm{hrs,} \mathrm{pain} \mathrm{scores,} \mathrm{nausea,} \mathrm{vomiting,}$ sedation and any other complications. Results: The time required for first analgesic demand was $3.9 \pm 0.8 \mathrm{hrs}$ in RQ group and $0.1 \pm 0.2 \mathrm{hrs}$ in group SQ which was statistically significant. The total paracetamol consumption in 24 hours was $1.2 \pm 0.4 \mathrm{gm}$ in group RQ and $2.9 \pm 0.7 \mathrm{gm}$ in group SQ , the difference being remarkable. Conclusion: Transmuscular quadratus lumborum block can produce quality analgesia after laparoscopic colorectal surgery. TQLB not only improves the visual analogue scale (VAS) score but also decreases the rescue analgesic consumption without any complications.
\end{abstract}

Keywords:- Transmuscular quadratus lumborum block, laparoscopic, colorectal surgery, postoperative analgesia.

\section{INTRODUCTION}

Though thoracic epidural was regarded as gold standard for postoperative pain control in various abdominal and thoracic surgeries, it is getting obsolete gradually.[1] As search for better alternative technique for postoperative analgesia goes on, abdominal blocks like transverse abdominal plane (TAP) block and transmuscular quadratus lumborum block
(TQLB) are continuously under investigation for postoperative analgesia. Earlier trials on TAP blocks have not shown any positive results as it only blocks somatic pains, without blocking visceral pain as local anesthetics fails to spread to the paravertebral space. [2] The TQLB can produce better analgesia after laparoscopic colorectal surgery. Local anesthetics block the nerves transmitting visceral pain in the thoracic paravertebral 
Annals of International Medical and Dental Research

E-ISSN: 2395-2822 | P-ISSN: 2395-2814

Vol-8, Issue-1 | January-February 2022

DOI: 10.53339/aimdr.2022.8.1.22

Page no- 168-174 | Section- Research Article (Anaesthesiology)

space which includes T4-L1 dermatomes. In TQLB, local anesthetics spread between the psoas major and the quadratus lumborum to the fascial interspace posterior to the transverse fascia. [3] TQLB was first described by Borglum et al and they concluded that TQLB could provide better postoperative analgesia. [4] In few cadaveric trials of the TQLB, dye was seen spreading in the paravertebral space which surrounds the somatic nerves, and the thoracic sympathetic trunk. [5] In literature few studies have concluded that TQLB can provide good postoperative analgesia in abdominal surgery. ${ }^{6,7]}$ As literature is silent on any data on TQLB in laparoscopic colorectal surgery we have done this trial to assess the efficacy of TQLB in colorectal surgery for postoperative analgesia.

\section{MATERIAL AND METHODS}

This is a prospective randomised double blind trial which was undertaken after approval from our Institutional ethics committee. Informed consent was obtained from all patients. Study was conducted during the period from Jan 2020 to Aug 2021. A total of 64 patients aged between 30- 70 years with ASA (I/II) posted for laparoscopic colorectal surgery under general anaesthesia were included in our study. Patients having history of allergy to local anaesthetics, coagulopathy,chronic renal failure,BMI $>25 \mathrm{~kg} / \mathrm{m} 2$, block site infection, and cardio respiratory diseases were excluded. All the patients were randomly split sequentially into group RQ and group SQ using a computer generated random numbers. The allocated sequence was put in sealed opaque envelopes and were opened in the operation theatre.
Group RQ received ropivacaine in TQLB and group SQ received saline in TQLB. Study drug was prepared in 2 syringes of $20 \mathrm{ml}$ containing either $40 \mathrm{ml}$ saline or $40 \mathrm{ml} 0.375 \%$ ropivacaine by an investigator who was blinded to the study. The anaesthesiologist who administered the blocks and recorded pain characteristics were blinded to the group allocation. In the operation theatre, IV access was established using an 18-gauge IV cannula and ringers lactate was started. Electrocardiograph (ECG), non- invasive blood pressure (NIBP) monitor, and pulse oximeter were attached and base line data were recorded. Ultrasound (US) guided TQLB was given as described by Jodan. ${ }^{8]}$ IV midazolam $0.03 \mathrm{mg} / \mathrm{kg}$ and fentanyl $1 \mathrm{mcg} / \mathrm{kg}$ was administered. Then all patients were put in the left and right lateral decubitus position and block was given. A low-frequency $\quad 18-6 \mathrm{MHz}$ curvilinear ultrasound transducer was put just above the anterior and posterior iliac crest and below the rib cage. Structures like the transverse process, vertebral body, erector spinae muscle, quadratus lumborum muscle, and the psoas muscle making up the "shamrock" sign was identified.[8] Tip of the needle was directed between the psoas muscle and the quadratus lumborum muscle and $20 \mathrm{ml}$ of either of $0.375 \%$ ropivacaine or normal saline was injected into the interfascial plane on each side as per the allocated group. All the patients were tested for successful block and patients with successful block were allowed for surgery.

Anaesthesia was induced by IV injection of propofol $2 \mathrm{mg} / \mathrm{kg}$, fentanyl $1 \mathrm{mcg} / \mathrm{kg}$, and vecuronium $\quad 0.1 \mathrm{mg} / \mathrm{kg}$.Endotracheal intubation was done and anaesthesia was 
Annals of International Medical and Dental Research

E-ISSN: 2395-2822 | P-ISSN: 2395-2814

Vol-8, Issue-1 | January-February 2022

DOI: $10.53339 /$ aimdr.2022.8.1.22

Page no- 168-174 | Section- Research Article (Anaesthesiology)

maintained with a mixture of oxygen, nitrous oxide and isoflurane and end-tidal carbon dioxide level of 35-40 mm Hg was maintained. The isoflurane concentration was adjusted to keep the blood pressure and heart rate values within $20 \%$ of the preoperative measurements. At the end of surgery patients were extubated and were shifted to the postanaesthesia care unit. The pain was measured by the Visual Analogue Score (VAS) on the scale of 0- 10 (0 no pain and 10 - worst imaginable pain).[9] Complications like pruritus, sedation, nausea, and haemodynamic abnormalities were assessed in both the groups at 20min, 40min, 1hrs, 3hrs, 6hrs, 9hrs, 12hrs, 15hrs, 18hrs, $21 \mathrm{hrs}$ and 24 hours after the surgery. Rescue analgesia (iv paracetamol15mg/kg) was given when VAS score $>4$.Severity of PONV was measured on the 4 - point score (0 - absent, 1 mild, 2 - moderate and 3 -severe or vomiting). Patients were evaluated for the level of sedation by using a 4- point sedation scale (1awake,2- response to verbal command, 3 response to touch, 4 - deeply sedated and response to pain). Primary outcome was the time required for first analgesic request. Secondary outcome were total dose of paracetamol required in 24 hours, VAS scores, and any other complications. Sample size was calculated after doing a pilot study. Assuming $50 \%$ increase in the time of request for analgesia request as significant, keeping the power of the study at $80 \%$ and significance level of $95 \%$, a total of 28 subjects were required in each group. Keeping in mind about possible dropouts we have included 32 patients in each group. Continuous data was analysed for normality using the "Kolmogorov-Smirnov test" of normality. Normal distributed data was represented as mean \pm SD and was assessed using the student's $t$-test. Non-normally distributed data was analysed using the Mann- Whitney $\mathrm{U}$ - test. A $\mathrm{P}$ value $<0.05$ was considered significant.

\section{RESULTS}

A total of 70 patients posted for colorectal surgery were included in the study. 6 patients were excluded for not meeting inclusion criteria. 64 patients were enrolled in the study, out of which 2 patients of each group were excluded again from analysis as they were converted to open surgery. [Figure 1] The demographic profile, block performing time and duration of surgery were comparable in both the groups. [Table 1] The mean time to first analgesic request $3.9 \pm 0.8 \mathrm{hrs}$ hours in group RQ and $0.1 \pm 0.2$ hours in group SQ. $(\mathrm{P}<$ 0.05) The total dose of paracetamol consumed in 24 hours was1.2 $\pm 0.4 \mathrm{gm}$ in group RQ and $2.9 \pm 0.7 \mathrm{gm}$ in group SQ. $(\mathrm{P}<0.05)$ [Table 2]. At different time intervals, VAS scores were significantly lower in the Group RQ compared to the group SQ. $(p<0.05)$ [Table 3] There was no remarkable difference regarding $\mathrm{PONV}$ scores, and sedation between the two groups [Table 4].

Table 1: Descriptive variables of groups.

\begin{tabular}{|l|l|l|l|}
\hline Variables & Group RQ (n=30) & Group SQ (n=30) & P value \\
\hline Age (years) & $54.13 \pm 15.65$ & $53.78 \pm 14.98$ & 0.147 \\
\hline Female: Male $(\mathrm{n})$ & $26: 4$ & $26: 4$ & 0.179 \\
\hline
\end{tabular}


Annals of International Medical and Dental Research

E-ISSN: 2395-2822 | P-ISSN: 2395-2814

Vol-8, Issue-1 | January-February 2022

DOI: $10.53339 /$ aimdr.2022.8.1.22

Page no- 168-174 | Section- Research Article (Anaesthesiology)

\begin{tabular}{|l|l|l|l|}
\hline ASA I/II (n) & $19 / 11$ & $18 / 12$ & 0.163 \\
\hline Surgical time (mins) & $98.55 \pm 20.25$ & $97.9 \pm 21.34$ & 0.152 \\
\hline Block performing time (mins) & $7.34 \pm 1.84$ & $7.28 \pm 1.82$ & 0.159 \\
\hline BMI (kg/m2) & $22.14 \pm 3.36$ & $21.25 \pm 4.16$ & 0.148 \\
\hline
\end{tabular}

Values expressed as Mean \pm SD and No, SD: Standard deviation. Student's t test and Chi square test applied. P $<0.05$ is significant

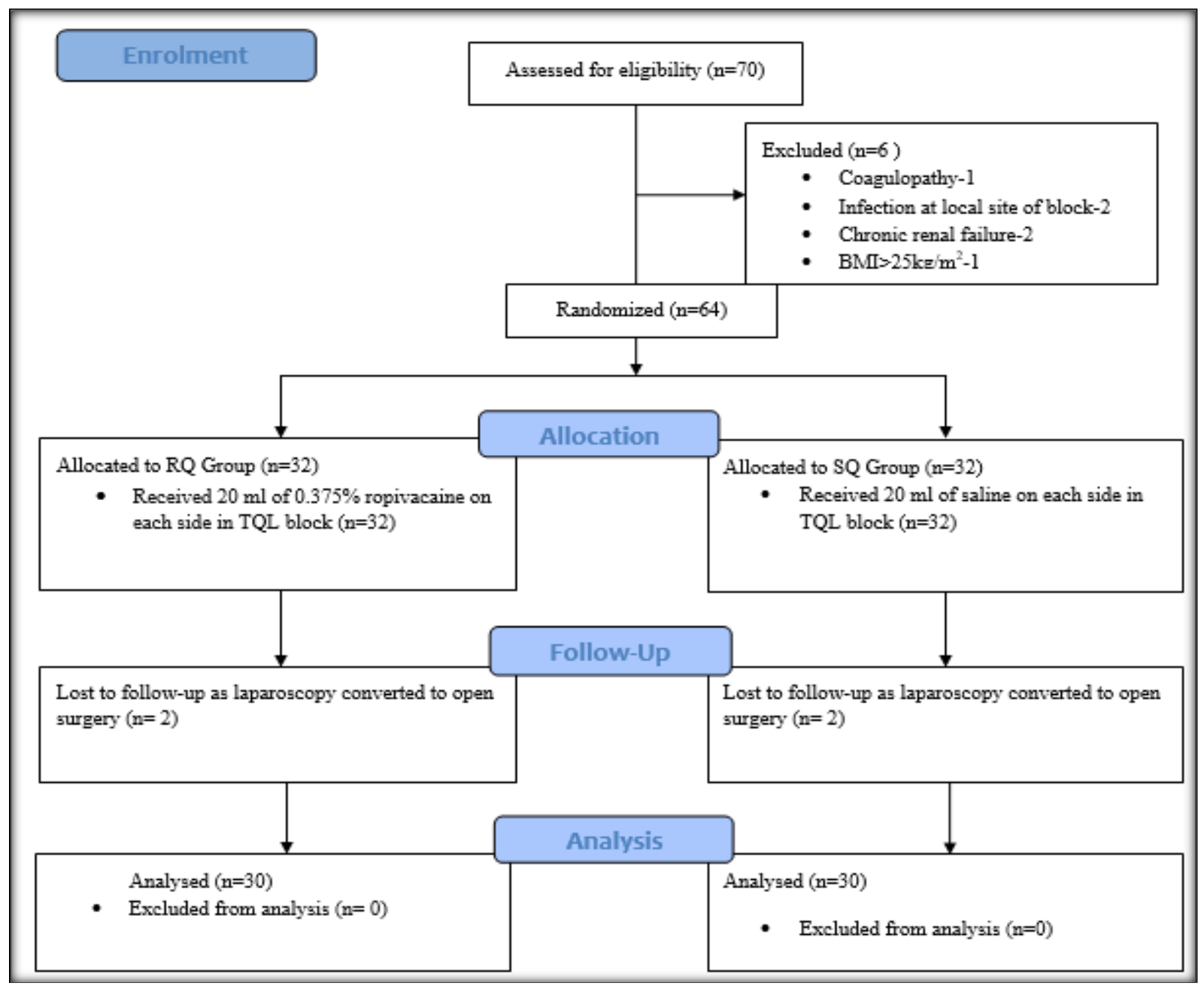

Figure 1: flow chart of the study

Table 2: Total analgesic consumption in $24 \mathrm{hrs}$ and time to first analgesia request

\begin{tabular}{|l|l|l|l|}
\hline Parameters & Group RQ (n=30 ) & Group SQ (n=30) & P value \\
\hline $\begin{array}{l}\text { Total analgesic consumption } \\
\text { (Paracetamol in gm) }\end{array}$ & $1.2 \pm 0.4$ & $3.9 \pm 0.8$ & 0.023 \\
\hline $\begin{array}{l}\text { Time to 1st rescue analgesia } \\
\text { request (hrs) }\end{array}$ & $3.9 \pm 0.8 \mathrm{hrs}$ & $0.1 \pm 0.2$ & 0.021 \\
\hline
\end{tabular}


Annals of International Medical and Dental Research

E-ISSN: 2395-2822 | P-ISSN: 2395-2814

Vol-8, Issue-1 | January-February 2022

DOI: $10.53339 /$ aimdr.2022.8.1.22

Page no- 168-174 | Section- Research Article (Anaesthesiology)

Values expressed as Mean \pm SD, SD: Standard deviation. Student's $t$ test and Chi square test applied. $\mathrm{P}<0.05$ is significant

Table 3: Post-operative VAS scores

\begin{tabular}{|l|l|l|l|}
\hline Times of Measurement & Group RQ $(\mathbf{n}=\mathbf{3 0})$ & Group SQ $(\mathbf{n}=\mathbf{3 0})$ & P value \\
\hline 20th $\min$ & $1.21 \pm 2.16$ & $3.4 \pm 2.55$ & 0.016 \\
\hline 40th $\min$ & $1.87 \pm 2.15$ & $3.25 \pm 1.98$ & 0.018 \\
\hline 1st h & $1.98 \pm 1.9$ & $3.19 \pm 1.85$ & 0.021 \\
\hline 3rd h & $1.94 \pm 1.5$ & $3.18 \pm 1.58$ & 0.023 \\
\hline 6th $\mathrm{h}$ & $1.88 \pm 1.5$ & $3.28 \pm 1.73$ & 0.021 \\
\hline 9th $\mathrm{h}$ & $1.94 \pm 1.67$ & $3.75 \pm 1.59$ & 0.012 \\
\hline 12th $\mathrm{h}$ & $2.2 \pm 1.8$ & $3.35 \pm 1.28$ & 0.031 \\
\hline 15th $\mathrm{h}$ & $2.1 \pm 1.9$ & $3.38 \pm 1.28$ & 0.015 \\
\hline 18th $\mathrm{h}$ & $2.4 \pm 1.6$ & $3.85 \pm 1.74$ & 0.016 \\
\hline 21st h & $2.34 \pm 1.5$ & $3.18 \pm 1.63$ & 0.014 \\
\hline 24th h & $2.95 \pm 0.94$ & $3.35 \pm 1.25$ & 0.011 \\
\hline
\end{tabular}

Values expressed as Mean \pm SD, SD: Standard deviation.

Table 4: Incidence of PONV and sedation

\begin{tabular}{|l|l|l|l|}
\hline & Group RQ $(\mathbf{n}=\mathbf{3 0})$ & Group SQ $(\mathbf{n}=\mathbf{3 0})$ & P value \\
\hline Nausea & 4 & 3 & 0.561 \\
\hline Vomiting & 2 & 3 & 0.472 \\
\hline Sedation & 3 & 2 & 0.481 \\
\hline
\end{tabular}

\section{DISCUSSION}

In our study we found that TQLB produced efficient pain relief compared to saline group. Patients in the group RQ had remarkably delayed the time for first analgesia request, reduced the analgesia requirement in 24 hours and produced lower VAS scores compared to group SQ. Abdominal wall blocks like TAP block have been used for postoperative analgesia in different abdominal surgeries. But there is controversy regarding its efficacy as the number of studies with poor efficacy is increasing.[10,11,12] Most of the abdominal wall blocks, like TAP and fascia transversalis block, only affect somatic pain, not visceral pain. Spread of the local anaesthetic to the paravertebral space is mandatory for controlling visceral pain. In TQLB, the local anaesthetic spreads between psoas major and quadratus lumborum, which block the ventral rami of the spinal nerve thereby controlling visceral pain. [13,14] Abduallah, et al,[15] in their study opined that the use of TQLB decreased post-operative analgesic consumption and post-operative pain score in patients undergoing total hip replacement. Xia et al,[16] in their study found that the combined TQLB and fascia iliaca compartment block produced prolonged postoperative analgesia after total hip arthroplasty. He et al,[17] in their study concluded that ultrasound-guided TQLB provided efficient postoperative analgesia after total hip arthroplasty.Zhu et al,[18] in their study opined that the ultrasound-guided TQLB produced efficient postoperative analgesia in 
Annals of International Medical and Dental Research

E-ISSN: 2395-2822 | P-ISSN: 2395-2814

Vol-8, Issue-1 | January-February 2022

DOI: $10.53339 /$ aimdr.2022.8.1.22

Page no- 168-174 | Section- Research Article (Anaesthesiology)

patients undergoing laparoscopic nephrectomy and it reduced the consumption of opoids postoperatively. Jadon et al,[19] opined that the ultrasound-guided TQLB provided prolonged and effective postoperative analgesia after laparoscopic hysterectomy surgery. It not only reduced the fentanyl consumption but also improved the visual analogue scale (VAS) score postoperatively. Coppens et al, [20] in their study opined that TQLB provided efficient postoperative analgesia when compared to controlled intravenous analgesia with morphine alone.In their study by Deng et al,[21] they found that the TQLB provided better postoperative analgesia in comparison to transverses abdominal plane block in patients

\section{REFERENCES}

1. Levy BF, Scott MJ, Fawcett W, Fry C, Rockall TA. Randomized clinical trial of epidural, spinal or patient-controlled analgesia for patients undergoing laparoscopic colorectal surgery. $\mathrm{Br} \mathrm{J}$ Surg. 2011;98(8):1068-78. doi: 10.1002/bjs.7545.

2. Smith SR, Draganic B, Pockney P, Holz P, Holmes R, Mcmanus B, Carroll R. Transversus abdominis plane blockade in laparoscopic colorectal surgery: a doubleblind randomized clinical trial. Int J Colorectal Dis. 2015;30(9):1237-45. doi: 10.1007/s00384-015-2286-7.

3. Dewinter G, Coppens S, Van de Velde M, D'Hoore A, Wolthuis A, Cuypers E, et al. Quadratus Lumborum Block Versus Perioperative Intravenous Lidocaine for Postoperative Pain Control in Patients Undergoing Laparoscopic Colorectal Surgery: A Prospective, Randomized, Double-blind Controlled Clinical Trial. Ann Surg. 2018;268(5):769-775. doi: 10.1097/SLA.0000000000002888.

4. Gopal TVS. Ultrasound-guided transmuscular quadratus lumborum plane catheters: In the plane or out of it?. Indian J Anaesth. 2019;63(8):609-610. doi:10.4103/ija.IJA_585_19

5. Dam M, Moriggl B, Hansen CK, Hoermann R, Bendtsen TF, Børglum J. The Pathway of Injectate Spread With the Transmuscular Quadratus undergoing laparoscopic colorectal surgery. Though all above studies were in agreement with our study, we suggest further large scale studies to validate our study findings.

\section{CONCLUSIONS}

Ultrasound-guided TQLB with ropivacaine provides efficient postoperative analgesia and prolongs the first request of analgesia in patients undergoing laparoscopic colorectal surgery. It reduces the VAS score and total analgesic consumption without any complications in post operative period.

Lumborum Block: A Cadaver Study. Anesth Analg. 2017;125(1):303-312. doi: 10.1213/ ANE.0000000000001922.

6. Dam M, Hansen CK, Poulsen TD, Azawi NH, Wolmarans M, Chan V, et al. Transmuscular quadratus lumborum block for percutaneous nephrolithotomy reduces opioid consumption and speeds ambulation and discharge from hospital: a single centre randomised controlled trial. $\mathrm{Br} \mathrm{J}$ Anaesth. 2019;123(2):e350-e358. doi: 10.1016/j.bja.2019.04.054.

7. Steingrímsdóttir GE, Hansen CK, Børglum J. Ultrasound-guided transmuscular quadratus lumborum catheters for elective caesarean section: A protocol for a single-centre, double-blind randomised trial. Acta Anaesthesiol Scand. 2020;64(8):1218-1223. doi: 10.1111/aas.13601.

8. Andersen EB, Tanggaard K, Nielsen MV, Hansen CK, Dam M, Poulsen TD, et al. Ultrasound-guided transmuscular quadratus lumborum block catheter technique. Anaesthesia. 2020;75(3):412-413. doi: 10.1111/anae.14955.

9. Rawal N. Current issues in postoperative pain management. Eur J Anaesthesiol. 2016;33(3):160-71. doi: 10.1097/EJA.0000000000000366.

10.Walter CJ, Maxwell-Armstrong C, Pinkney TD, Conaghan PJ, Bedforth N, Gornall CB, et al. A 
Annals of International Medical and Dental Research

E-ISSN: 2395-2822 | P-ISSN: 2395-2814

Vol-8, Issue-1 | January-February 2022

DOI: 10.53339/aimdr.2022.8.1.22

Page no- 168-174 | Section- Research Article (Anaesthesiology)

randomised controlled trial of the efficacy of ultrasound-guided transversus abdominis plane (TAP) block in laparoscopic colorectal surgery. Surg Endosc. 2013;27(7):2366-72. doi: 10.1007/s00464-0132791-0.

11. Coppens S, Rex S, Fieuws S, Neyrinck A, D'Hoore A, Dewinter G. Transmuscular quadratus lumborum (TQL) block for laparoscopic colorectal surgery: study protocol for a double-blind, prospective randomized placebo-controlled trial. Trials. 2020;21(1):581. doi:10.1186/s13063-020-04525-6

12. Oh TK, Yim J, Kim J, Eom W, Lee SA, Park SC, et al. Effects of preoperative ultrasound-guided transversus abdominis plane block on pain after laparoscopic surgery for colorectal cancer: a doubleblind randomized controlled trial. Surg Endosc. 2017;31(1):127-134. doi: 10.1007/s00464-016-4941-7.

13.Dam M, Moriggl B, Hansen CK, Hoermann R, Bendtsen TF, Børglum J. The Pathway of Injectate Spread With the Transmuscular Quadratus Lumborum Block: A Cadaver Study. Anesth Analg. 2017;125(1):303-312. doi: 10.1213/ANE.0000000000001922.

14.Saranteas T, Anagnostopoulos D, Kostroglou A, Souvatzoglou R. The "Shamrock method" for ultrasound-guided lumbar plexus nerve block in the supine position. J Clin Anesth. 2021;71:110249. doi: 10.1016/j.jclinane.2021.110249.

15. Abduallah MA, Ahmed SA, Abdelghany MS. The effect of post-operative ultrasound-guided transmuscular quadratus lumborum block on postoperative analgesia after hip arthroplasty in elderly patients: A randomised controlled double-blind study. Indian J Anaesth. 2020;64(10):887-893. doi: 10.4103/ija.IJA_275_20.

16.Xia Q, Ding W, Lin C, Xia J, Xu Y, Jia M. Postoperative pain treatment with transmuscular quadratus lumborum block and fascia iliaca compartment block in patients undergoing total hip arthroplasty: a randomized controlled trial. BMC Anesthesiol. 2021;21(1):188. doi: 10.1186/s12871-02101413-7.

17.He J, Zhang L, He WY, et al. Ultrasound-Guided Transmuscular Quadratus Lumborum Block Reduces Postoperative Pain Intensity in Patients Undergoing Total Hip Arthroplasty: A Randomized, DoubleBlind, Placebo-Controlled Trial. Pain Res Manag. 2020;2020:1035182. doi:10.1155/2020/1035182

18.Zhu M, Qi Y, He H, Lou J, Pei Q, Mei Y. Analgesic effect of the ultrasound-guided subcostal approach to transmuscular quadratus lumborum block in patients undergoing laparoscopic nephrectomy: a randomized controlled trial. BMC Anesthesiol. 2019;19(1):154. doi: 10.1186/s12871-019-0825-4.

19. Jadon A, Ahmad A, Sahoo RK, Sinha N, Chakraborty S, Bakshi A. Efficacy of transmuscular quadratus lumborum block in the multimodal regimen for postoperative analgesia after total laparoscopic hysterectomy: A prospective randomised doubleblinded study. Indian J Anaesth. 2021;65(5):362-368. doi: 10.4103/ija.IJA_1258_20.

20. Coppens S, Rex S, Fieuws S, Neyrinck A, D'Hoore A, Dewinter G. Transmuscular quadratus lumborum (TQL) block for laparoscopic colorectal surgery: study protocol for a double-blind, prospective randomized placebo-controlled trial. Trials. 2020;21(1):581.

21.Deng $\mathrm{W}$, Long X, Li M, et al. Quadratus lumborum block versus transversus abdominis plane block for postoperative pain management after laparoscopic colorectal surgery: A randomized controlled trial. Medicine (Baltimore). 2019;98(52):e18448.

Source of Support: Nil, Conflict of Interest: None declared 